PROCEEDINGS OF THE

AMERICAN MATHEMATICAL SOCIETY

Volume 137, Number 4, April 2009, Pages 1467-1478

S 0002-9939(08)09748-7

Article electronically published on November 18,2008

\title{
ON THE DISCRETE HEAT EQUATION TAKING VALUES ON A TREE
}

\author{
CARL MUELLER AND KIJUNG LEE
}

(Communicated by Richard C. Bradley)

\begin{abstract}
This paper was motivated by the question of studying PDE or stochastic PDE taking values on nonsmooth spaces. This is a hard problem in general, so we concentrate on a test case: the heat equation taking values on the union of rays emanating from the origin. We construct a series of discrete approximation to the solution and show that they converge to a limit. Unfortunately, we do not know if the limit is uniqueness. Our tools are probabilistic, exploiting the well-known connection between Brownian motion and the heat equation.
\end{abstract}

\section{INTRODUCTION}

The basic ideas about differential equations taking values on manifolds are well known. Usually one localizes to a coordinate patch and studies the differential equation using local coordinates. If the manifold structure is smooth, then this procedure yields a new differential equation on Euclidean space, and one can use existing theory.

However, there are two cases which may cause trouble: the manifold may not be smooth, or the equation may have stochastic terms which lead to singularities. We will not say much about the stochastic case, although it was the motivation for this work. The reader can consult Funaki [Fun83, where the author studies the heat equation with additive noise taking values on a manifold. He requires the noise to be very smooth in $x$, excluding the interesting case of white noise. For the white noise case, the problem is still unsolved.

Turning to the deterministic case, there has been some work PDEs on nonsmooth manifolds. For example, Eells and Fuglede EF01 deal with harmonic maps between Riemannian polyhedra. For the most part, though, not much is known about this situation, and the reasons are not hard to discover. Traditional PDE theory completely relies on linear techniques. For example, $u+v$ makes no sense if $u, v$ take values in a manifold, and for the same reason we cannot integrate manifold-valued functions or define Sobolev spaces like $L_{p}$ or $W_{p}^{k}$ in the usual way. This is why one sets up coordinate patches which have a linear structure.

Received by the editors January 9, 2008.

2000 Mathematics Subject Classification. Primary 60H15; Secondary 35R60, 35K05.

Key words and phrases. Heat equation, white noise, stochastic partial differential equations.

The first author was supported by NSF and NSA grants.

(C)2008 American Mathematical Society Reverts to public domain 28 years from publication 
As for a physical application, the stochastic heat equation with solutions taking values in $\mathbb{R}^{3}$ is one of the main models used in polymer science; see Doi and Edwards, DE88. Thus, we can think of $u(t, x)$ as the position of an elastic string at time $t$ and at length $x$ along the string. In some situations the string might be confined to a region or surface, so we are led to consider the heat equation taking values on a manifold.

This paper has a modest goal. We consider a very simple nonsmooth manifold, the union of rays emanating from the origin, and consider the heat equation taking values in this space. We find a sequence of discrete approximations, and show sequential compactness using the Arzela-Ascoli theorem. Our main tool is a probabilistic representation of solutions. Unfortunately, we do not prove uniqueness, but our guess is that there is a unique limit for such sequences. It would also be nice to define our equation in the generalized sense, so we could say that our limit point satisfies the equation. These questions are unsolved at the moment.

This paper is organized in the following way. In section 2 we set up our heat equation and present our notation. In section 3 we consider discrete approximations and suggest a tool for sequential compactness. After giving some estimates in section 4, we prove our main theorem in section 5. which states that our sequence of discrete approximations is sequentially compact.

Here are some general references. For the heat equation and other PDEs, see Evans [Eva98]. For probability, random walks and martingales, see Durrett Dur96]. Finally, we list some commonly known notation.

$\mathbb{N}$ : the set of natural numbers.

$\mathbb{Z}$ : the set of integers.

$\mathbb{R}$ : the set of real numbers.

$\mathbb{R}_{+}$: the set of nonnegative numbers.

$E X$ : the mathematical expectation of a random variable $X$.

$x \wedge y:$ the minimum of $\{x, y\}$.

$\mathbf{1}_{A}$ : indicator or characteristic function on event set $A$.

$N\left(\mu, \sigma^{2}\right)$ : normal distribution with mean $\mu$ and variance $\sigma^{2}$.

\section{Notation}

We wish to consider solutions $u(t, x)$ with $t>0$ and $x \in \mathbb{R}$ to the heat equation

$$
\frac{\partial u}{\partial t}=\frac{1}{2} \frac{\partial^{2} u}{\partial x^{2}}
$$

taking values in a tree, which we define below.

For the range of $u(t, x)$, we consider a tree $\mathcal{T}$ embedded in $\mathbb{R}^{2}$. Let $\mathcal{T}$ be the union of $N$ rays which meet at one common point $(0,0)$. To put it concretely, we can set

$$
\mathcal{T}=\bigcup_{i=1}^{N}\left\{\left(r, \theta_{i}\right): r \geq 0\right\}
$$

with a finite number $N$ of distinct angles $\theta_{i}$ in polar coordinates.

Note that for such a tree $\mathcal{T}$, we can define a metric $\rho(a, b)$ between two points $a, b \in \mathcal{T}$ as the length of the shortest path (geodesic) in $\mathcal{T}$ from $a$ to $b$. In particular, the triangle inequality holds:

$$
\rho(a, b) \leq \rho(a, c)+\rho(c, b)
$$


for any $a, b, c \in \mathcal{T}$. Note that there is a unique such geodesic joining $a$ and $b$, and a unique midpoint along the geodesic. We denote this midpoint as

$$
a \odot b
$$

and we use this key notion throughout the paper.

\section{The Discrete heAT Equations}

Let us consider $u_{0}: \mathbb{R} \rightarrow \mathcal{T}$, which serves as our initial condition.

Given a positive integer $n$ we define a grid $T_{n}$ on $[0, \infty)$ with spacing $\frac{1}{n}$, and a grid $R_{n}$ on $\mathbb{R}$ with spacing $\frac{1}{\sqrt{n}}$, i.e.

$$
\begin{aligned}
T_{n} & =\left\{t_{i}=\frac{i}{n} \in[0, \infty): i \in \mathbb{N} \cup\{0\}\right\}, \\
R_{n} & =\left\{x_{j}=\frac{j}{\sqrt{n}} \in \mathbb{R}: j \in \mathbb{Z}\right\} .
\end{aligned}
$$

Next, we define a grid $\mathbf{L}_{n}$ containing every other point of $T_{n} \times R_{n}$ :

$$
\mathbf{L}_{n}=\left\{\left(\frac{i}{n}, \frac{j}{\sqrt{n}}\right) \in T_{n} \times R_{n}: i+j \text { is even }\right\} .
$$

We define the discrete heat equation on $\mathbf{L}_{n}$. We are interested in finding the solution $u_{n}=u_{n}\left(t_{i}, x_{j}\right),\left(t_{i}, x_{j}\right) \in \mathbf{L}_{n}$, satisfying

$$
\begin{aligned}
u_{n}\left(t_{i+1}, x_{j}\right) & =u_{n}\left(t_{i}, x_{j+1}\right) \odot u_{n}\left(t_{i}, x_{j-1}\right), \\
u_{n}\left(0, x_{j}\right) & =u_{0}\left(x_{j}\right) .
\end{aligned}
$$

This is our discrete approximation for a given $n$. Equation (3.1) is just the analogue of the discrete approximation to the heat equation taking values in $\mathbb{R}$ :

$$
\begin{aligned}
\frac{\partial v}{\partial t} & =\frac{1}{2} \frac{\partial^{2} v}{\partial x^{2}}, \\
v(0, x) & =v_{0}(x) .
\end{aligned}
$$

Indeed, for $\left(t_{i}, x_{j}\right) \in \mathbf{L}_{n}$ we could approximate (3.2) by the Euler method:

$$
\begin{aligned}
\frac{\left[v_{n}\left(t_{i+1}, x_{j}\right)-v_{n}\left(t_{i}, x_{j}\right)\right]}{\frac{1}{n}} & =\frac{1}{2} \frac{\left[v_{n}\left(t_{i}, x_{j+1}\right)-2 v_{n}\left(t_{i}, x_{j}\right)+v_{n}\left(t_{i}, x_{j-1}\right)\right]}{\left(\frac{1}{\sqrt{n}}\right)^{2}}, \\
v_{n}\left(0, x_{j}\right) & =v_{0}\left(x_{j}\right),
\end{aligned}
$$

which reduces to (3.1) with $v_{n}$ in place of $u_{n}$.

The equation (3.1) allows us to find the solution $u_{n}\left(t_{i}, x_{j}\right)$ by induction on time $t_{i}$. To define $u_{n}(t, x)$ for all $t \geq 0, x \in \mathbb{R}$, we use linear interpolation as follows. First fix $t_{i}$ and define $u\left(t_{i}, x\right)$ for $x \in \mathbb{R}$ by linear interpolation. Next, fix $x \in \mathbb{R}$ and define $u(t, x)$ for $t \geq 0$ by linear interpolation.

We conjecture that $u_{n}, n=1,2, \ldots$ are good approximations to the solution satisfying (2.1) in a natural sense. So, our question is how to show that the approximations are reliable.

Let $\left\{X_{i}: i=0,1,2, \ldots\right\}$ be a simple random walk on the space grid $R_{n}$ with $X_{0}$ at a given grid point. This means that the step size of the walk is $\frac{1}{\sqrt{n}}$, and the time 
between steps is $\frac{1}{n}$. Using this random walk, we can give a probabilistic solution to (3.3) as follows:

$$
v_{n}\left(t_{i}, x_{j}\right)=E_{x_{j}}\left[v_{0}\left(X_{i}\right)\right]=\sum_{y_{k} \in R_{n}} v_{0}\left(y_{k}\right) P\left(X_{i}=y_{k}\right),
$$

where $E_{x_{j}}$ means the expectation given $X_{0}=x_{j}$. One can use this expression for showing sequential compactness of $\left\{v_{n}\right\}$. Our goal is to carry over this reasoning to the heat equation with values in $\mathcal{T}$. However, $\mathcal{T}$ is not a linear space, so the summation in (3.4) is not well-defined in $\mathcal{T}$ and we suffer from this difficulty. However, if we use coupling, a probabilistic tool whose motivation comes from the random walk, and keep track of the entire history of the random walks, then we can overcome the difficulty.

\section{Preliminary estimates}

For this section, fix $n$. We will consider two simple random walks $X^{(i)}, i=1,2$, taking values in $R_{n}$. Thus, each random walk has steps of size $\frac{1}{\sqrt{n}}$ and time between steps $\frac{1}{n}$. We assume that $X_{0}^{(i)}=x_{i}$ with $\left(0, x_{i}\right) \in \mathbf{L}_{n}$ for $i=1,2$. We note that with probability $1,\left(t_{k}, X_{k}^{(i)}\right) \in \mathbf{L}_{n}$ for $i, j=1,2$ and $k=0,1, \ldots$.

We are interested in two simple random walks which are coupled; we will use the coupling later in Section 5. Recall that a coupling of the random walks is a realization of $X^{(i)}, i=1,2$ on a single probability space such that the marginal distribution of each walk $X^{(i)}$ is the same as before.

Suppose the random walks $X^{(i)}$ are coupled in such a way that for a fixed $k$ we have $X_{k}^{(1)}=X_{k}^{(2)}$ with high probability. Then it seems reasonable that $E_{x_{1}}\left[v_{0}\left(X_{k}^{(1)}\right)\right]$ and $E_{x_{2}}\left[v_{0}\left(X_{k}^{(2)}\right)\right]$ should be close. Since this kind of coupling is easier if $x_{1}$ and $x_{2}$ are close, we might use coupling to show that solutions $v(t, x)$ of (3.2), which are given by (3.4), are regular in $x$. This reasoning has been used before; see Cranston [Cra91, for example. Such regularity helps to prove the sequential compactness of solutions.

Lemma 4.1. There is a coupling of the random walks $X^{(i)}, i=1,2$ and a constant $C>0$ not depending on $n$ such that for all $k \in \mathbb{N}$ we have

$$
P\left(X_{k}^{(1)} \neq X_{k}^{(2)}\right) \leq\left[C\left(\frac{n}{k}\right)^{1 / 2}\left|x_{1}-x_{2}\right|\right] \wedge 1 .
$$

Proof. 1. Let $h=\left|x_{1}-x_{2}\right| / 2$. Translating if necessary, we may assume that $x_{1}=h$, $x_{2}=-h$. Construct $X^{(1)}$ on some probability space $(\Omega, \mathcal{F}, P)$ in the usual way. Let $\tau$ be the first time $k$ that $X_{k}^{(1)}=0$. Define $X^{(2)}$ as follows:

$$
X_{k}^{(2)}= \begin{cases}-X_{k}^{(1)} & \text { if } k<\tau, \\ X_{k}^{(1)} & \text { if } k \geq \tau,\end{cases}
$$

so that with probability 1 , the two walks meet eventually. This coupling is well known, and the reader can easily verify that $X^{(2)}$ has the correct marginal distribution.

2. If $x_{1}=x_{2}$, then $X^{(1)}$ is identical with $X^{(2)}$ and our claim holds trivially. Hence, let us assume $x_{1} \neq x_{2}$. Since we assumed that $\left(0, x_{i}\right) \in \mathbf{L}_{n}$ for $i=1,2$, we 
note that $\left|x_{1}-x_{2}\right| \geq \frac{2}{\sqrt{n}}$, or $h \sqrt{n} \geq 1$. We use this fact to get (4.2) below from the previous line.

Using the reflection principle in the usual way (see Feller [Fel68], Chapter III), we get

$$
\begin{aligned}
P\left(X_{k}^{(1)} \neq X_{k}^{(2)}\right) & =P(\tau>k) \\
& =1-P\left(\inf _{0 \leq i \leq k} X_{i}^{(1)} \leq 0\right) \\
& =1-P\left(X_{k}^{(1)} \leq 0\right)-P\left(X_{k}^{(1)}<0\right) \\
& \leq P\left(\left|X_{k}^{(1)}-X_{0}^{(1)}\right| \leq h\right) .
\end{aligned}
$$

Heuristically, one can easily finish the proof using the normal approximation, but we will give a rigorous argument using Stirling's approximation. We assume that $k=2 m$ is even, leaving the odd case to the reader. Let $S_{k}$ be the simple random walk with steps of size 1 starting at $S_{0}=0$. Note that the probability function $p_{i}=P\left(S_{k}=i\right)$ is even. $p_{i}$ is zero at every other site $i$, and where it is nonzero it is radially nonincreasing. Therefore,

$$
\begin{aligned}
P\left(\left|X_{k}^{(1)}-X_{0}^{(1)}\right| \leq h\right) & =P\left(\left|S_{2 m}\right| \leq h \sqrt{n}\right) \\
& \leq(2 h \sqrt{n}+1) P\left(S_{2 m}=0\right) \\
& \leq 3 h \sqrt{n} P\left(S_{2 m}=0\right) .
\end{aligned}
$$

Next, Stirling's formula (see, for instance, (7.3.4) on p. 211 of [Chu79]) gives us the following:

$$
\begin{aligned}
P\left(S_{2 m}=0\right) & =\left(\begin{array}{c}
2 m \\
m
\end{array}\right) 2^{-2 m} \\
& \leq \frac{e^{\frac{1}{12 \cdot 2 m}}}{e^{\frac{1}{12(m+1 / 2)}} \cdot 2} \cdot \frac{\sqrt{2 \pi 2 m} e^{-2 m}(2 m)^{2 m}}{\left(\sqrt{2 \pi m} e^{-m} m^{m}\right)^{2}} \cdot 2^{-2 m} \\
& =\exp \left[\frac{1}{24} \frac{\frac{1}{2}-3 m}{m\left(m+\frac{1}{2}\right)}\right] \cdot(\pi m)^{-1 / 2} \\
& \leq \sqrt{\frac{2}{m}}
\end{aligned}
$$

and so

$$
P\left(S_{k}=0\right) \leq \frac{1}{\sqrt{k}}
$$

We can finish the proof of Lemma 4.1 by putting together (4.1), (4.2), and (4.3), remembering that $h=\left|x_{1}-x_{2}\right| / 2$, and choosing $C \geq \frac{3}{2}$.

Now we state a lemma about midpoints in $\mathcal{T}$.

Lemma 4.2. Let $x_{1}, x_{2}, y_{2}, y_{2} \in \mathcal{T}$. Then

$$
\rho\left(x_{1} \odot x_{2}, y_{1} \odot y_{2}\right) \leq \frac{\rho\left(x_{1}, y_{1}\right)}{2}+\frac{\rho\left(x_{2}, y_{2}\right)}{2} .
$$


Proof. There are several cases according to the locations of $x_{1}, x_{2}, y_{1}, y_{2}$ in rays.

1. If $x_{1}, x_{2}, y_{1}, y_{2}$ lie on just 1 or 2 rays, then (4.4) follows from the triangle inequality on $\mathbb{R}_{+}$or $\mathbb{R}$.

2. Suppose that $x_{1}, x_{2}, y_{1}, y_{2}$ lie on 3 rays and they do not lie on just 1 or 2 rays. Then one ray must contain 2 points, and the other 2 rays contain 1 point each. By symmetry, we can reduce to two situations. Let us denote three rays of $\mathcal{T}$ by $R_{i}, i=1,2,3$.

Case 1. Suppose that $x_{1}, x_{2}$ lie on a common ray $R_{3}, y_{1}$ lies on ray $R_{1}$, and $y_{2}$ lies on ray $R_{2}$. Let $y_{1}^{\prime} \in R_{2}$ be the same distance from the origin as $y_{1}$. Then $\rho\left(x_{1}, y_{1}\right)=\rho\left(x_{1}, y_{1}^{\prime}\right)$. However, it is easily seen that

$$
\rho\left(0, y_{1} \odot y_{2}\right) \leq \rho\left(0, y_{1}^{\prime} \odot y_{2}\right)
$$

and that

$$
\rho\left(x_{1} \odot x_{2}, y_{1} \odot y_{2}\right)=\rho\left(x_{1} \odot x_{2}, 0\right)+\rho\left(0, y_{1} \odot y_{2}\right) .
$$

Thus, since $x_{1}, x_{2}, y_{1}^{\prime}, y_{2}$ lie on 2 rays, we conclude that

$$
\begin{aligned}
\rho\left(x_{1} \odot x_{2}, y_{1} \odot y_{2}\right) & =\rho\left(x_{1} \odot x_{2}, 0\right)+\rho\left(0, y_{1} \odot y_{2}\right) \\
& \leq \rho\left(x_{1} \odot x_{2}, 0\right)+\rho\left(0, y_{1}^{\prime} \odot y_{2}\right) \\
& =\rho\left(x_{1} \odot x_{2}, y_{1}^{\prime} \odot y_{2}\right) \\
& \leq \frac{\rho\left(x_{1}, y_{1}^{\prime}\right)}{2}+\frac{\rho\left(x_{2}, y_{2}\right)}{2} \\
& =\frac{\rho\left(x_{1}, y_{1}\right)}{2}+\frac{\rho\left(x_{2}, y_{2}\right)}{2} .
\end{aligned}
$$

Case 2. Suppose that $x_{1}, y_{1}$ lie on a common ray $R_{3}, x_{2}$ lies on ray $R_{1}$, and $y_{2}$ lies on ray $R_{2}$.

First suppose that either $x_{1} \odot x_{2}$ or $y_{1} \odot y_{2}$ lie on $R_{3}$. By changing the labeling, we may assume $x_{1} \odot x_{2}$ lies on $R_{3}$. Let $y_{2}^{\prime} \in R_{1}$ be the same distance from the origin as $y_{2}$. Replacing $y_{2}$ by $y_{2}^{\prime}$, we see that the left side of (4.4) has not changed, but the right side has become smaller, and all 4 points lie on just 2 rays. Thus, (4.4) follows from the triangle inequality on $\mathbb{R}$.

Secondly, suppose that neither $x_{1} \odot x_{2}$ nor $y_{1} \odot y_{2}$ lie on $R_{3}$. Thus, $x_{1} \odot x_{2} \in R_{1}$ and $y_{1} \odot y_{2} \in R_{2}$. Also, suppose without loss of generality that $x_{1}$ lies closer to the origin than $y_{1}$. One can see that moving $y_{1}$ to position $x_{1}$ changes $\rho\left(0, y_{1} \odot y_{2}\right)$ and $\frac{\rho\left(x_{1}, y_{1}\right)}{2}$ by the same amount. Hence, we have

$$
\begin{aligned}
\rho\left(x_{1} \odot x_{2}, y_{1} \odot y_{2}\right)-\frac{\rho\left(x_{1}, y_{1}\right)}{2} & =\rho\left(0, x_{1} \odot x_{2}\right)+\rho\left(0, y_{1} \odot y_{2}\right)-\frac{\rho\left(x_{1}, y_{1}\right)}{2} \\
& =\rho\left(0, x_{1} \odot x_{2}\right)+\rho\left(0, x_{1} \odot y_{2}\right) \\
& \leq \frac{\rho\left(0, x_{2}\right)}{2}+\frac{\rho\left(0, y_{2}\right)}{2} \\
& =\frac{\rho\left(x_{2}, y_{2}\right)}{2} .
\end{aligned}
$$


3. Finally, let's assume that $x_{1}, x_{2}, y_{1}, y_{2}$ lie on 4 rays and none of them shares the same ray with the others. In this case, we clearly see

$$
\begin{aligned}
\rho\left(x_{1} \odot x_{2}, y_{1} \odot y_{2}\right) & \leq \rho\left(x_{1}, 0\right) \wedge \rho\left(x_{2}, 0\right)+\rho\left(y_{1}, 0\right) \wedge \rho\left(y_{2}, 0\right) \\
& \leq \frac{\rho\left(x_{1}, 0\right)+\rho\left(x_{2}, 0\right)}{2}+\frac{\rho\left(y_{1}, 0\right)+\rho\left(y_{2}, 0\right)}{2} \\
& =\frac{\rho\left(x_{1}, 0\right)+\rho\left(y_{1}, 0\right)}{2}+\frac{\rho\left(x_{2}, 0\right)+\rho\left(y_{2}, 0\right)}{2} \\
& =\frac{\rho\left(x_{1}, y_{1}\right)}{2}+\frac{\rho\left(x_{2}, y_{2}\right)}{2} .
\end{aligned}
$$

This finishes the proof of Lemma 4.2.

\section{Sequential compactness}

Here we prove our main theorem, which indicates that the process of our discrete approximations is stable. In the theorem, our condition on $u_{0}$ is certainly not the weakest possible (see Assumption 5.2), but it yields a simple proof.

First, we need some definitions and an assumption on $u_{0}$. Since $\rho$ is a metric on $\mathcal{T}$, we can speak of uniform continuity and equicontinuity for functions taking values in $\mathcal{T}$.

Definition 5.1. If a positive function $\zeta$ is defined on positive real numbers $(0, \infty)$ and satisfies $\lim _{h \rightarrow 0^{+}} \zeta(h)=0$, then we write $\zeta \in \mathcal{M}_{0}$.

Assumption 5.2. We assume that $u_{0}$ is uniformly continuous with a modulus function $\kappa$. That is, $\kappa \in \mathcal{M}_{0}$ is monotone increasing and for all $x, y \in \mathbb{R}$ we have

$$
\rho\left(u_{0}(x), u_{0}(y)\right) \leq \kappa(|x-y|) .
$$

We also assume that the growth of $\kappa$ is no faster than linear:

$$
\kappa(h) \leq N_{0}+N_{1} h
$$

for some absolute constants $N_{0}, N_{1}>0$.

We state our main theorem.

Theorem 5.3. Let $u_{0}: \mathbb{R} \rightarrow \mathcal{T}$ satisfy Assumption 5.2, Then the collection of solutions $\left\{u_{n}: n=1,2, \ldots\right\}$ to (3.1) is sequentially compact in the topology of uniform convergence on compact subsets of $[0, \infty) \times \mathbb{R}$.

According to the Arzela-Ascoli theorem for the functions taking values on a metric space, we need only show that on each compact subset of $K \subset[0, \infty) \times \mathbb{R}$, the family $\left\{u_{n}\right\}$ is equicontinuous. In fact, we will show that for any $K$ of the form $K=[0, a] \times[-b, b]$,

$$
\rho\left(u_{n}(t, x), u_{n}(s, y)\right) \leq \zeta(|(t, x)-(s, y)|), \quad(t, x),(s, y) \in K
$$

for a function $\zeta \in \mathcal{M}_{0}$ not depending on $n$.

The following two lemmas are the main ingredients in the proof of Theorem 5.3 .

Lemma 5.4. For given $K=[0, a] \times[-b, b]$ with $a, b>0$ there exists a function $\zeta_{1} \in \mathcal{M}_{0}$ which does not depend on $n, t$ and satisfying

$$
\rho\left(u_{n}(t, x), u_{n}(t, y)\right) \leq \zeta_{1}(|x-y|), \quad(t, x),(t, y) \in K
$$


Remark 5.5. The reader can check that the proof of Lemma 5.4 even works with $K$ of the form $[0, a] \times \mathbb{R}$.

Proof. 1. Fix $t=t_{I} \in T_{n} \cap[0, a]$ and $x, y \in R_{n} \cap[-b, b]$. We are using the two random walks $X^{(i)}$ defined on a probability space $(\Omega, \mathcal{F}, P)$ from Lemma 4.1 such that

Let

$$
X_{0}^{(1)}=x, \quad X_{0}^{(2)}=y .
$$

$$
M_{k}=\rho\left(u_{n}\left(t_{I}-t_{k}, X_{k}^{(1)}\right), u_{n}\left(t_{I}-t_{k}, X_{k}^{(2)}\right)\right), \quad k=0, \ldots, I
$$

and let $\mathcal{F}_{k}$ be the $\sigma$-field generated by $\left\{X_{m}^{(1)}, X_{m}^{(2)}: m \leq k\right\}$. We claim that $\left(M_{k}, \mathcal{F}_{k}\right)$ is a submartingale.

Fix an event set $A$ which belongs to the partition of $\Omega$ which generates $\mathcal{F}_{k}$. Naturally, $X_{k}^{(1)}, X_{k}^{(2)}$, and, hence, $M_{k}$ are constants on $A$. Let $X_{k}^{(1)}=\alpha, X_{k}^{(2)}=$ $\beta, M_{k}=\gamma$ on $A$.

Case 1. $\alpha=\beta$.

In this case $X_{k+1}^{(1)}-X_{k}^{(1)}=X_{k+1}^{(2)}-X_{k}^{(2)}$ on $A$ by the construction of $X^{(2)}$. The set $A$ can be divided into $A^{+}$and $A^{-}$according to the value of $X_{k+1}^{(1)}-X_{k}^{(1)}$ :

$$
A^{+}=A \cap\left\{X_{k+1}^{(1)}-X_{k}^{(1)}=\frac{1}{\sqrt{n}}\right\} \quad A^{-}=A \cap\left\{X_{k+1}^{(1)}-X_{k}^{(1)}=-\frac{1}{\sqrt{n}}\right\} .
$$

It is easy to see that the conditional probabilities $P\left(A^{+} \mid A\right), P\left(A^{-} \mid A\right)$ are $\frac{1}{2}$.

By our discrete heat equation (3.1), on $A$ we have

$$
\begin{array}{r}
M_{k}=\gamma=\rho\left(u_{n}\left(t_{I}-t_{k+1}, \alpha+\frac{1}{\sqrt{n}}\right) \odot u_{n}\left(t_{I}-t_{k+1}, \alpha-\frac{1}{\sqrt{n}}\right),\right. \\
\left.u_{n}\left(t_{I}-t_{k+1}, \beta+\frac{1}{\sqrt{n}}\right) \odot u_{n}\left(t_{I}-t_{k+1}, \beta-\frac{1}{\sqrt{n}}\right)\right)
\end{array}
$$

for $k=0, \ldots, I-1$ and by Lemma 4.2 ,

$$
\begin{aligned}
\gamma \leq & \frac{1}{2} \rho\left(u_{n}\left(t_{I}-t_{k+1}, \alpha+\frac{1}{\sqrt{n}}\right), u_{n}\left(t_{I}-t_{k+1}, \beta+\frac{1}{\sqrt{n}}\right)\right) \\
& +\frac{1}{2} \rho\left(u_{n}\left(t_{I}-t_{k+1}, \alpha-\frac{1}{\sqrt{n}}\right), u_{n}\left(t_{I}-t_{k+1}, \beta-\frac{1}{\sqrt{n}}\right)\right) .
\end{aligned}
$$

This implies

$$
\begin{aligned}
M_{k} & \leq \frac{E\left[M_{k+1} I_{A^{+}}\right]}{P(A)}+\frac{E\left[M_{k+1} I_{A^{-}}\right]}{P(A)} \\
& =E\left[M_{k} \mid \mathcal{F}_{k}\right]
\end{aligned}
$$

on $A$.

Case 2. $\alpha \neq \beta$.

This case is similar to the previous case. The differences are the following. This time we have $X_{k+1}^{(1)}-X_{k}^{(1)}=-\left(X_{k+1}^{(2)}-X_{k}^{(2)}\right)$ on $A$ and we use

$$
\begin{aligned}
\gamma \leq & \frac{1}{2} \rho\left(u_{n}\left(t_{I}-t_{k+1}, \alpha+\frac{1}{\sqrt{n}}\right), u_{n}\left(t_{I}-t_{k+1}, \beta-\frac{1}{\sqrt{n}}\right)\right) \\
& +\frac{1}{2} \rho\left(u_{n}\left(t_{I}-t_{k+1}, \alpha-\frac{1}{\sqrt{n}}\right), u_{n}\left(t_{I}-t_{k+1}, \beta+\frac{1}{\sqrt{n}}\right)\right),
\end{aligned}
$$


which again follows (5.4) and Lemma 4.2 with commutativity of $\odot$. The rest for reaching the inequality (5.5) is essentially the same.

Since we chose arbitrary $A$ in the partition, $\left(M_{k}, \mathcal{F}_{k}\right)$ is, indeed, a submartingale. It follows that

$$
M_{0} \leq E\left[M_{I}\right] .
$$

2. Therefore, for $t=t_{I} \in T_{n} \cap[0, a]$ and $x, y \in R_{n} \cap[-b, b]$,

$$
\begin{aligned}
\rho\left(u_{n}(t, x), u_{n}(t, y)\right) \leq & E\left[\rho\left(u_{0}\left(X_{I}^{(1)}\right), u_{0}\left(X_{I}^{(2)}\right)\right)\right] \\
\leq & \left.\rho\left(u_{0}(x), u_{0}(y)\right)+E\left[\rho\left(u_{0}\left(X_{I}^{(1)}\right), u_{0}(x)\right)\right) \mathbf{1}_{\left\{X_{I}^{(1)} \neq X_{I}^{(2)}\right\}}\right] \\
& +E\left[\rho\left(u_{0}(y), u_{0}\left(X_{I}^{(2)}\right)\right) \mathbf{1}_{\left\{X_{I}^{(1)} \neq X_{I}^{(2)}\right\}}\right] \\
= & (I)+(I I)+(I I I)
\end{aligned}
$$

by the triangle inequality. Let $h=|x-y|$. First note that by (5.1),

$$
(I) \leq \kappa(h) .
$$

Next, since $(I I)$ and $(I I I)$ are similar, we will only estimate $(I I)$. Again by (5.1),

$$
(I I) \leq E\left[\kappa\left(\left|X_{I}^{(1)}-x\right|\right) \mathbf{1}_{\left\{X_{I}^{(1)} \neq X_{I}^{(2)}\right\}}\right]=:(I I b) .
$$

Recall that our coupling estimate in Lemma 4.1 implies that

$$
P\left(X_{I}^{(1)} \neq X_{I}^{(2)}\right) \leq\left[C t^{-1 / 2}|x-y|\right] \wedge 1 .
$$

Roughly speaking, if $t=t_{I}$ is small, then $\kappa\left(\left|X_{I}^{(1)}-x\right|\right)$ will be small. If $t$ is not small, then we can use (5.5). Choose a constant $\gamma \in(0,1)$.

Case 1. $t \leq h^{2-2 \gamma}=|x-y|^{2-2 \gamma}$.

We have

$$
\begin{aligned}
(I I b) & =E\left[\kappa\left(\left|X_{I}^{(1)}-x\right|\right) \mathbf{1}_{\left\{X_{I}^{(1)} \neq X_{I}^{(2)}\right\}}\right] \\
& \leq E\left[\kappa\left(\left|X_{I}^{(1)}-x\right|\right)\right] \\
& \leq \xi(h)
\end{aligned}
$$

with $\xi \in \mathcal{M}_{0}$. To show that the final line holds with $\xi$ not depending on $n$, we prove the following: For any constant $\alpha \in\left(0, \frac{1}{2}\right)$,

$$
E\left[\kappa\left(\left|X_{I}^{(1)}-x\right|\right)\right] \leq \kappa\left(t^{\frac{1}{2}-\alpha}\right)+N_{0} t^{2 \alpha}+N_{1} t^{1 / 2}=: \delta(t)
$$

holds where the constants $N_{0}, N_{1}$ come from the linear growth condition of $\kappa$. We recall that the walk $X^{(1)}$ is designed after the choice of $n$ and $x$. We note that 
$X^{(1)}-x$ doesn't depend on $x$ and $\delta(\cdot)$ itself in (5.6) works for all $n$. We observe

$$
\begin{aligned}
E\left[\kappa\left(\left|X_{I}^{(1)}-x\right|\right)\right]= & \sum_{-t n \leq j \leq t n} \kappa\left(\frac{|j|}{\sqrt{n}}\right) P\left(X_{I}^{(1)}-x=\frac{j}{\sqrt{n}}\right) \\
= & \sum_{|j|<t^{1 / 2-\alpha} \sqrt{n}} \kappa\left(\frac{|j|}{\sqrt{n}}\right) P\left(\frac{S_{t n}}{\sqrt{t n}}=\frac{j}{\sqrt{t n}}\right) \\
& +\sum_{|j| \geq t^{1 / 2-\alpha} \sqrt{n}} \kappa\left(\frac{|j|}{\sqrt{n}}\right) P\left(\frac{S_{t n}}{\sqrt{t n}}=\frac{j}{\sqrt{t n}}\right) \\
= & \sigma_{1}+\sigma_{2},
\end{aligned}
$$

where $S$. is a simple random walk with the step size 1 . Obviously, $\sigma_{1} \leq \kappa\left(t^{\frac{1}{2}-\alpha}\right)$. Meanwhile, we have

$$
\begin{aligned}
\sigma_{2} & \leq N_{0} P\left(\left|\frac{S_{t n}}{\sqrt{t n}}\right| \geq \frac{1}{t^{\alpha}}\right)+N_{1} \sqrt{t} E\left|\frac{S_{t n}}{\sqrt{t n}}\right| \\
& \leq N_{0} t^{2 \alpha} \cdot E\left|\frac{S_{t n}}{\sqrt{t n}}\right|^{2}+N_{1} \sqrt{t} \cdot\left(E\left|\frac{S_{t n}}{\sqrt{t n}}\right|^{2}\right)^{1 / 2} \\
& \leq N_{0} t^{2 \alpha}+N_{1} \sqrt{t}
\end{aligned}
$$

since the variance of $\frac{S_{t n}}{\sqrt{t n}}$ is 1 . Hence, (5.6) holds. We set $\xi(h)=\delta\left(h^{2-2 \gamma}\right)$ in the situation $t \leq h^{2-2 \gamma}$.

Case 2. $t \geq h^{2-2 \gamma}=|x-y|^{2-2 \gamma}$.

Using (5.2), we find

$$
\begin{aligned}
(I I b) & \leq E\left[\left(N_{0}+N_{1}\left|X_{t}^{(1)}-x\right|\right) \mathbf{1}_{\left\{X_{t}^{(1)} \neq X_{t}^{(2)}\right\}}\right] \\
& =N_{0} P\left(X_{t}^{(1)} \neq X_{t}^{(2)}\right)+N_{1} E\left[\left|X_{t}^{(1)}-x\right| \mathbf{1}_{\left\{X_{t}^{(1)} \neq X_{t}^{(2)}\right\}}\right] .
\end{aligned}
$$

Now (5.5) states that

$$
P\left(X_{t}^{(1)} \neq X_{t}^{(2)}\right) \leq C t^{-1 / 2}|x-y| \text {. }
$$

Using the Cauchy-Schwarz inequality and redefining $C$ if necessary, we get

$$
\begin{aligned}
E\left[\left|X_{t}^{(1)}-x\right| \mathbf{1}_{\left\{X_{t}^{(1)} \neq X_{t}^{(2)}\right\}}\right] & \leq\left(E\left|X_{t}^{(1)}-x\right|^{2}\right)^{1 / 2} P\left(X_{t}^{(1)} \neq X_{t}^{(2)}\right)^{1 / 2} \\
& =C t^{1 / 2}\left(t^{-1 / 2}|x-y|\right)^{1 / 2} .
\end{aligned}
$$

Putting together (5.7), (5.8), and (5.9), we conclude that

$$
\begin{aligned}
(I I b) & \leq C\left[t^{-1 / 2}|x-y|+\left(t^{-1 / 2}|x-y|\right)^{1 / 2}\right] \\
& \leq C\left(h^{\gamma}+h^{\gamma / 2}\right),
\end{aligned}
$$

where $C$ may depend on $a$, the bound of $K$ in the time direction, but it does not depend on $n, x, y$.

3. We note that in our continuity argument it is enough to consider $u_{n}$ on $(t, x) \in$ $\left(T_{n} \times R_{n}\right) \cap K$ since we construct $u_{n}$ outside of $T_{n} \times R_{n}$ by linear interpolation. We conclude that there exists a function $\zeta_{1}$ satisfying our claim. 
Lemma 5.6. There exists a function $\zeta_{2} \in \mathcal{M}_{0}$ which does not depend on $n, x$ and satisfies

$$
\left.\rho\left(u_{n}(t, x), u_{n}(s, x)\right)\right) \leq \zeta_{2}(|t-s|), \quad t, s \geq 0, x \in \mathbb{R}
$$

Proof. 1. Translating if necessary, we may assume that $s=0, x=0$.

2. Fix $t_{I} \in T_{n}$ and consider a random walk $X$. with $X_{0}=0$ as in the beginning of section 4 . We denote the probability space by $(\Omega, \mathcal{F}, P)$. Let

$$
Z_{k}=\rho\left(u_{n}\left(t-t_{k}, X_{k}\right), u(0,0)\right), \quad k=0, \ldots, I
$$

and let $\mathcal{F}_{k}$ be the $\sigma$-field generated by $\left\{X_{m}: m \leq k\right\}$. We claim that $\left(Z_{k}, \mathcal{F}_{k}\right)$ is a submartingale. As in the discussion of the submartingale $M_{k}$, fix an event set $A$ which belongs to the partition of $\Omega$ which generates $\mathcal{F}_{k}$. Let $X_{k}=\alpha, Z_{k}=\gamma$ on $A$ and define

$$
A^{+}=A \cap\left\{X_{k+1}-X_{k}=\frac{1}{\sqrt{n}}\right\} \quad A^{-}=A \cap\left\{X_{k+1}-X_{k}=-\frac{1}{\sqrt{n}}\right\},
$$

where we note that the conditional probabilities $P\left(A^{+} \mid A\right), P\left(A^{-} \mid A\right)$ are $\frac{1}{2}$. On $A$ we have

$$
\begin{aligned}
Z_{k}=\gamma=\rho\left(u_{n}\left(t_{I}-t_{k+1}, \alpha+\frac{1}{\sqrt{n}}\right) \odot u_{n}\left(t_{I}-t_{k+1}, \alpha-\frac{1}{\sqrt{n}}\right)\right. & \\
& \left.u_{n}(0,0) \odot u_{n}(0,0)\right)
\end{aligned}
$$

for $k=0, \ldots, I-1$ and by Lemma 4.2 ,

$$
\begin{aligned}
\gamma \leq & \frac{1}{2} \rho\left(u_{n}\left(t_{I}-t_{k+1}, \alpha+\frac{1}{\sqrt{n}}\right), u_{n}(0,0)\right) \\
& +\frac{1}{2} \rho\left(u_{n}\left(t_{I}-t_{k+1}, \alpha-\frac{1}{\sqrt{n}}\right), u_{n}(0,0)\right),
\end{aligned}
$$

which implies

$$
\begin{aligned}
Z_{k} & \leq \frac{E\left[Z_{k+1} I_{A^{+}}\right]}{P(A)}+\frac{E\left[Z_{k+1} I_{A^{-}}\right]}{P(A)} \\
& =E\left[Z_{k} \mid \mathcal{F}_{k}\right]
\end{aligned}
$$

on $A$. Since we chose $A$ arbitrary in the partition, $\left(Z_{k}, \mathcal{F}_{k}\right)$ is a submartingale and we have

$$
Z_{0} \leq E\left[Z_{I}\right]
$$

3. Hence, for $t=t_{I} \in T_{n}$ we get

$$
\begin{aligned}
\rho\left(u_{n}(t, 0), u_{n}(0,0)\right) & \leq E\left[\rho\left(u_{0}\left(X_{I}\right), u_{0}(0)\right)\right] \\
& \leq E\left[\kappa\left(X_{I}\right)\right] \\
& \leq \delta(t),
\end{aligned}
$$

with $\delta(\cdot)$ in the proof of Lemma 5.4. The choice of $\zeta_{2}$ is easy and the lemma is proved.

We are ready to prove Theorem 5.3 . 
Proof of Theorem [5.3. We continue with the comment following the statement of Theorem 5.3, Observing

$$
\rho\left(u_{n}(t, x), u_{n}(s, y)\right) \leq \rho\left(u_{n}(t, x), u_{n}(t, y)\right)+\rho\left(u_{n}(t, y), u_{n}(s, y)\right)
$$

and using Lemmas 5.4 and 5.6, we have (5.3) with $\zeta=\zeta_{1}+\zeta_{2}$. Theorem 5.3 follows.

\section{ACKNOWLEDGMENT}

The beginning of this paper came from discussions the authors had near the nice espresso machine on the 8th floor of the Hylan Building, University of Rochester, during the second author's stay.

\section{REFERENCES}

[Chu79] Kai Lai Chung. Elementary probability theory with stochastic processes. Third edition. Undergraduate Texts in Mathematics. Springer-Verlag, New York-Heidelberg, 1979. MR.560506 (81k:60002)

[Cra91] Michael Cranston. Gradient estimates on manifolds using coupling. J. Funct. Anal., 99(1):110-124, 1991. MR 1120916 (93a:58175)

[DE88] M. Doi and S.F. Edwards. The theory of polymer dynamics, volume 73 of The International Series of Monographs in Physics. Oxford University Press, Oxford, 1988.

[Dur96] Richard Durrett.Probability: Theory and examples. Duxbury Press, Belmont, CA, second edition, 1996. MR $1609153(98 \mathrm{~m}: 60001)$

[EF01] J. Eells and B. Fuglede. Harmonic maps between Riemannian polyhedra, volume 142 of Cambridge Tracts in Mathematics. Cambridge University Press, Cambridge, 2001. With a preface by M. Gromov. MR 1848068 (2002h:58017)

[Eva98] Lawrence C. Evans. Partial differential equations, volume 19 of Graduate Studies in Mathematics. American Mathematical Society, Providence, RI, 1998. MR.1625845 (99e:35001)

[Fel68] William Feller. An introduction to probability theory and its applications. Vol. I. Third edition. John Wiley \& Sons Inc., New York, 1968. MR0228020 (37:3604)

[Fun83] Tadahisa Funaki. Random motion of strings and related stochastic evolution equations. Nagoya Math. J., 89:129-193, 1983. MR692348 (85g:60063)

Department of Mathematics, University of Rochester, Rochester, New York 14627

E-mail address: cmlr@math.rochester.edu

Department of Mathematics, Yonsei University, Seoul 120-749, Republic of Korea

E-mail address: kijung@yonsei.ac.kr 